\title{
The Cosmic Origins Spectrograph and the Future of Ultraviolet Astronomy
}

\author{
J. Michael Shull \\ University of Colorado, CASA, Department of Astrophysical \& Planetary Sciences, 391-UCB, \\ Boulder, CO 80309
}

\begin{abstract}
I describe the capabilities of the Cosmic Origins Spectrograph, scheduled for May 2009 installation on the Hubble Space Telescope. With a factor-of-ten increase in far-UV throughput for moderate-resolution spectroscopy, COS will enable a range of scientific programs that study hot stars, AGN, and gas in the interstellar medium, intergalactic medium, and galactic halos. We also plan a large-scale HST Spectroscopic Legacy Project for QSO absorption lines, galactic halos, and AGN outflows. Studies of next-generation telescopes for UV/O astronomy are now underway, including small, medium, and large missions to fill the imminent ten-year gap between the end of Hubble and a plausible launch of the next large mission. Selecting a strategy for achieving these goals will involve hard choices and tradeoffs in aperture, wavelength, and capability.
\end{abstract}

Keywords: UV astronomy

PACS: 98.62.Ra

\section{INTRODUCTION}

In the near term, the future of ultraviolet space astronomy is reasonably bright. The Galex satellite was well-ranked in NASA's recent senior review and will continue its programs of broad-band spectral imaging in the ultraviolet. For UV spectroscopy, the Space Shuttle is scheduled to install the Cosmic Origins Spectrograph (COS) on the Hubble Space Telescope (HST) in mid-2009. Although delayed, the COS far-UV (FUV) spectrograph will provide an order-of-magnitude increase in throughput for moderate resolution $\left(R \approx 20,000\right.$ or $\left.15 \mathrm{~km} \mathrm{~s}^{-1}\right)$ spectra between $1150-1775 \AA$.

However, beyond the lifetimes of Galex and HST/COS, there may be a hiatus in ultraviolet-optical (UV/O) space astronomy. Following a successful Hubble Servicing Mission 4 (May 2009), we anticipate an additional 5-10 years of Hubble lifetime. However, after mission completion of the two Great Observatories, Hubble and Chandra, we might expect a gap of perhaps 10 years in both UV/O and X-ray space astronomy until after 2025, when major new missions could be approved and built. In this talk, I will briefly describe some of the scientific projects enabled by COS, then turn to strategies for filling this 10-year gap with new missions in the UV/O spectral bands. Some key issues are:

- What should be the science drivers for new UV/O missions?

- What is the correct distribution of small, medium, and large missions?

- How should the UV/O community advocate the best science?

- What are tradeoffs in aperture, wavelength band, and other capabilities? 


\section{MAJOR SCIENCE GOALS FOR COS}

The Cosmic Origins Spectrograph (COS) was designed to provide high-throughput $\left(A_{\text {eff }}>2000 \mathrm{~cm}^{2}\right)$, moderate-resolution $(R \approx 20,000)$ point-source spectroscopy in the far-ultraviolet $(\lambda \geq 1150 \AA)$. This capability would provide a factor-of-ten increase in throughput over previous UV spectrographs on Hubble over the range 1150-1775 $\AA$. In addition, a near-ultraviolet (NUV) channel (1750-3200 ̊) was added, although with only a moderate improvement (factors of 2-3) in effective area over STIS. The capabilities and pre-launch performance of COS were described by Froning \& Green (2008), excerpted in Table 1 below. The proposed science objectives were given by Morse et al. (1998) and updated briefly in this section.

TABLE 1. COS Far-Ultraviolet Wavelength Modes

\begin{tabular}{llclc}
\hline Grating & $\begin{array}{l}\text { Wavelength } \\
\text { Coverage }(\AA)\end{array}$ & $\begin{array}{c}\text { Wavelength Range } \\
(\text { per expo, } \AA \text { ) }\end{array}$ & $\begin{array}{l}\text { Resolution } \\
(R=\lambda / \Delta \lambda)\end{array}$ & $\begin{array}{c}\text { Average Sensitivity } \\
\left.\text { cts/s/resl/[erg cm }{ }^{-2} \mathrm{~s}^{-1} \AA^{-1}\right]\end{array}$ \\
\hline G130M & $1150-1450$ & 300 & $20,000-24,000$ & $1 \times 10^{13}$ \\
G160M & $1405-1775$ & 375 & $20,000-24,000$ & $7 \times 10^{12}$ \\
G140L & $1230-2050$ & $>820$ & $2400-3500$ & $3 \times 10^{13}$ \\
\hline
\end{tabular}

The major science goals of the Guaranteed Time Observations (GTO) with COS are enabled by the ability to observe faint targets $(V \approx 17.5)$ at $15 \mathrm{~km} \mathrm{~s}^{-1}$ resolution in the ultraviolet. This allows COS to acquire spectra of active galactic nuclei (AGN), hot stars (OB stars, white dwarfs, cataclysmic variables, binaries) in the Milky Way, and O stars in neighboring galaxies. Planned observations also include studies of planets, comets, disks, and extra-solar planet transits. Although COS has no long-slit capability, it will be able to perform emission-line measurements of nebulae, supernova remnants, and starburst galaxies, although with degraded spectral resolution.

Many of the COS science programs involve acquiring absorption-line spectra of interstellar and intergalactic gas in the Milky Way and its halo, in intervening galaxies, and in the intergalactic medium (IGM). Approximately half the 552 GTO orbits are devoted to programs of IGM science that focus on high-level questions:

- How was the "Cosmic Web" of intergalactic gas formed and structured?

- Where are the "missing baryons" synthesized in the Big Bang?

- What processes determine the IGM phases in temperature and ionization?

- What is the chemical composition and spatial distribution of metals in the IGM?

The common theme of these studies is to understand the geometry and composition of the Cosmic Web (Figure 1). The intergalactic gas is predicted to consist of complex, multi-phase structures (Davé et al. 2001; Cen \& Ostriker 2006) formed by gravitational instability and influenced by feedback from star formation in galaxies (ionizing radiation, outflows, and metal transport). To conduct these investigations, the COS-GTO team formulated a set of observing projects, using 43 background AGN as targets to probe the IGM, as well as intervening galaxy halos and the Milky Way interstellar medium (ISM). These studies are grouped into four projects totalling 253 orbits. 


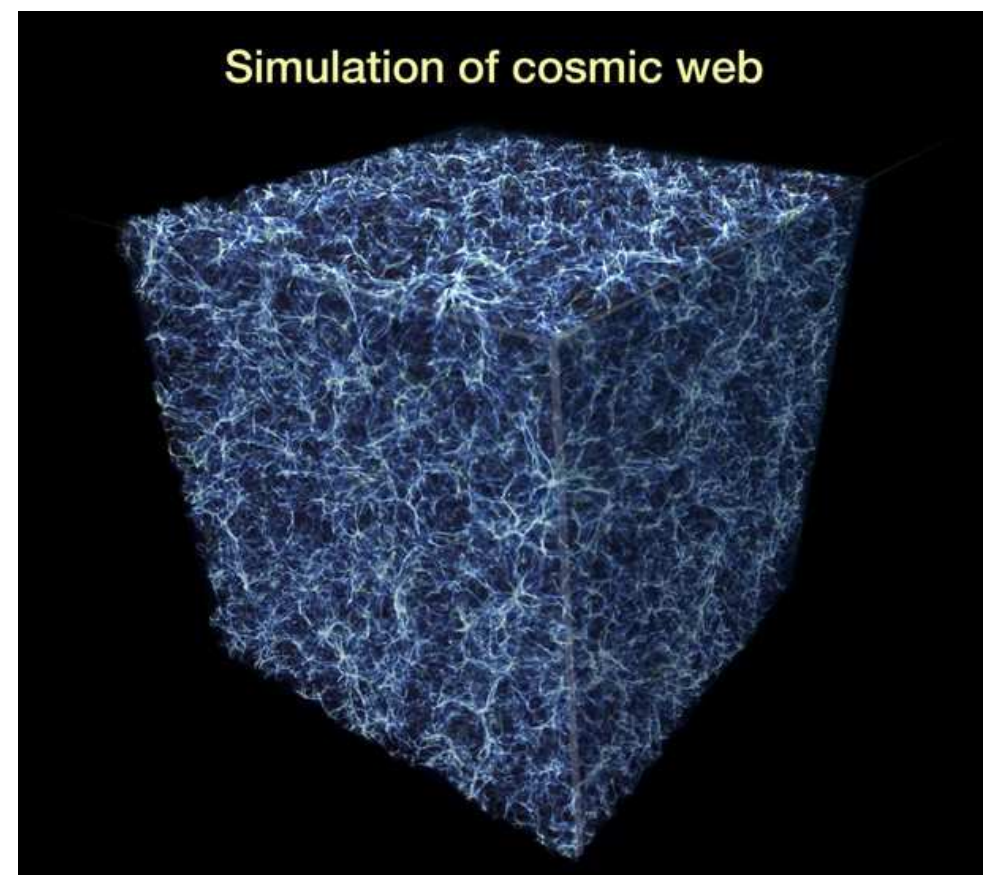

FIGURE 1. Distribution of baryons in the "Cosmic Web" at $z \approx 0.1$ from IGM simulations (Hallman et al. 2007). Large-scale structure develops in filaments, detectable (Danforth \& Shull 2008; Tripp et al. 2008) in both cold $\operatorname{Ly} \alpha$ absorbers and in shock-heated gas enriched to 1-10\% solar metallicity.

Large-Scale Structure in Baryons (100 orbits, 18 AGN). This program will study the IGM Ly $\alpha$ absorbers and associated metal-line systems, together with intervening galaxy halos and Galactic high velocity clouds (HVCs). Further analysis provides baryon content, ionization state, covering factor, velocity distribution, and metallicity.

Warm-Hot Intergalactic Medium (100 orbits, 17 AGN). Absorption studies of highly ionized gas (O VI, O V, O IV, N V, Ne VIII, etc) and broad Ly $\alpha$ lines in redshifted IGM absorbers out to $z \approx 0.7$. Many of these lines have rest wavelengths in the EUV, and enter the COS band at moderate redshifts (e.g., O VI $\lambda 1032,1038$ at $z \geq 0.12$ and $\mathrm{Ne}$ VIII $\lambda 770,780$ at $z \geq 0.48$ ).

Great Wall Tomography (19 orbits, 4 AGN). Four background AGN targets will be studied for IGM absorbers in common (or absent) to estimate their transverse sizes. Uncertainty in the characteristic size of the Ly $\alpha$ absorbers is a systematic error in determining their physical density $\left(n_{H}\right)$ and baryon content $\left(\Omega_{\mathrm{IGM}}\right)$.

He II Reionization Epoch (27 orbits, 4 AGN). The Ly $\alpha$ forest of He II (304 A rest frame) enters the COS band at $z \geq 2.78$. We will study He II (Gunn-Peterson) absorption toward two AGN, HE 2347-4342 and HS 1700+6416, studied previously by FUSE, but with higher signal-to-noise and velocity resolution of COS. We will compare these spectra with H I Ly $\alpha$ absorbers, probing the He II reionization process at $z \approx 3$. 
The GTO team has also developed several projects to study the gas content, ionization state, and metallicity of Galactic HVCs in the low Galactic halo. These studies will allow us to estimate the HVC contribution to the mass-infall rate to the disk and infer their nucleosynthetic origin (Collins, Shull, \& Giroux 2004, 2007).

\section{HST/COS COMMUNITY LEGACY PROJECT}

COS should make major discoveries in a range of astronomical areas. Because the COS/GTO team will devote only half its time to IGM studies, a comprehensive study of IGM evolution, galactic halos, and interactions of gas and galaxies will require considerably more observing time. With COS, astronomers have an opportunity to construct a Quasar Absorption Line Key Project, but with a far more powerful spectrograph than FOS, used for the first Hubble QSO Absorption-Line Key Project (e.g., Weymann et al. 1998). The IGM science can be pushed much farther in depth, resolution, and efficiency, using the 10-20 times higher FUV throughput and $15 \mathrm{~km} \mathrm{~s}^{-1}$ spectral resolution of COS, compared to $230 \mathrm{~km} \mathrm{~s}^{-1}$ with FOS. In concert with galaxy surveys out to $z=0.3$, we have the opportunity to study the IGM and its relationship with galaxies on a scale never before possible. Only at low redshifts will galaxy surveys reach to sufficiently low luminosity $\left(L \approx 0.1 L^{*}\right)$ to explore IGM connections with low-mass galaxies (Stocke et al. 2006).

\section{STScI Call for HST Legacy-Project White Papers}

In 2007, the Space Telescope Science Institute (STScI) issued a call for white papers, designed to study large-scale HST Legacy Projects that use new instruments (WFC3 and COS) to be installed in Servicing Mission 4. In December 2007, a group of us proposed a spectroscopic key project with COS, entitled Structure and Evolution of the Intergalactic Medium. We considered a three-year project of 500-1000 orbits, including supplements from Director's Discretionary Time. The project was designed to obtain a large number of QSO absorption lines: over 10,000 Ly $\alpha$ absorbers and corresponding metals lines, using 150-200 AGN as background targets and yielding moderate-resolution spectra at $\mathrm{S} / \mathrm{N} \approx 30$. This proposal was well received upon review at STScI, and we were encouraged to propose formally for a multi-year program during HST Cycles 18-20. In addition to the major study of the IGM, this program would address a range of topics and phenomena of broad community interest:

- Map the IGM large-scale structure at $z \leq 0.3$ compared to galaxy distribution

- Characterize the multi-phase baryon content of cold, warm, and hot IGM

- Derive connections between low- $z$ and high- $z$ IGM

- Measure the spatial transport and redshift-evolution of metallicity

- Measure the chemical extent of galactic halos and galaxy outflows

- Probe galactic feedback (energy, radiation, metals) into the IGM

- Measure HVC mass infall, covering factor, ionization, and metallicity

- Study quasar intrinsic absorption lines (AGN outflows, feedback to IGM) 


\section{Scientific Rationale for a COS Legacy Project}

The epoch at $z<1.5$ marks a time of rapid change in the star-formation rate over the last 9 Gyr of cosmic time, as galaxies co-evolved with the IGM. These interactions include clumpy infall, tidal flows, and galaxy winds that can pollute the intergalactic space with heavy elements from star formation. These feedback processes are essential to understanding the growth and regulation of galaxy sizes, masses, and metallicities. Cosmological simulations take great care to accurately represent gas flows in and out of galaxies. The IGM therefore provides a laboratory for testing the predictions of the thermal phases produced by large-scale structure formation and feedback.

Even at $z<0.1$, the majority of baryons still reside in the IGM, not in collapsed structures (galaxies, groups, clusters contain only 5-10\% of the baryons). UV spectra from HST and FUSE have identified approximately half in the Ly $\alpha$ forest (HI) absorbers and shock-heated $\left(10^{5}\right.$ to $\left.10^{6} \mathrm{~K}\right)$ IGM traced by O VI $(1032,1038 \AA)$ absorption lines. Studies of absorption in H I, He II, and heavy elements (multiple ions of C, N, O, Si, $\mathrm{Mg}, \mathrm{Fe}$ ) allow us to follow the evolution of baryons and metals from $z=0$ back to $z=6$.

As shown in Figure 2, key diagnostic UV lines are accessible only to space astronomy (satellite telescopes). With FUSE and STIS, we have reasonable measurements of the absorption-line frequency, $d N / d z$, of Ly $\alpha, \mathrm{O}$ VI, and six other metal ions at low redshifts: Si III $\lambda 1206$, Si IV $\lambda 1394$, C III $\lambda$ 977, C IV $\lambda 1549$, etc.) facilitate the photoionization corrections needed to derive gas metallicity. Detecting even hotter IGM gas requires X-ray absorbers (O VII, O VIII), but may come from studies (Savage et al. $2005)$ of the Ne VIII doublet (770, $780 \AA)$ which shifts into the COS band at $z>0.5$. Neon is abundant ( $\sim 20 \%$ of oxygen), and Ne VIII is sensitive to gas at $T \approx 10^{6} \mathrm{~K}$.

COS will be able to observe fainter AGN, thereby increasing the total redshift pathlength through the IGM out to $z \sim 0.7$. This will improve the statistics and allows us to probe the evolution of IGM phases (warm photoionized, warm-hot collisionally ionized gas). Access to fainter background targets also provides a higher spatial frequency of AGN on the sky, constraining the size and shape of Ly $\alpha$ absorbers, one of the systematic uncertainties in measuring $\Omega_{\mathrm{IGM}}$, the baryon content in the IGM. At redshifts $z<0.3$, we will be able to compare the large-scale structure in gas with that surveyed in galaxies (Sloan Survey, 2DF, 6dF). Obtaining a higher frequency of AGN sightlines on the sky will allow us to gauge how far metal pollution extends from galaxies.

COS spectra will have multiple uses, since the sight lines to AGN also probe outflows emanating from galactic nuclei, along with the ISM of intervening galaxies and Milky Way halo and disk. COS sight lines will produce rich spectra of interstellar matter, including infalling HVCs. This Legacy Project will be used by observers of ISM, IGM, and galaxy formation providing information about QSO continuum emission processes, nuclear winds, and the broad-line and narrow-line emission regions.

Other considerations include the usefulness of NUV spectra and the efficiency of large FUV wavelength coverage (out to $z=0.44$ in Ly $\alpha$ and $0.12<z<0.69$ in O VI). We also have the opportunity to obtain high-quality spectra $(\mathrm{S} / \mathrm{N}=30-40)$ spectra of the brightest targets. COS can observe fainter AGN targets at a higher spatial frequency, enabling "IGM tomography" of several filaments in the Cosmic Web. It can probe gas in the outer halos of galaxies and HVCs in our own Galaxy. With greater total redshift pathlength $(\Delta z \gg 10)$ we may find stronger Ly $\alpha$ absorbers (partial Lyman-Limit systems, even a 


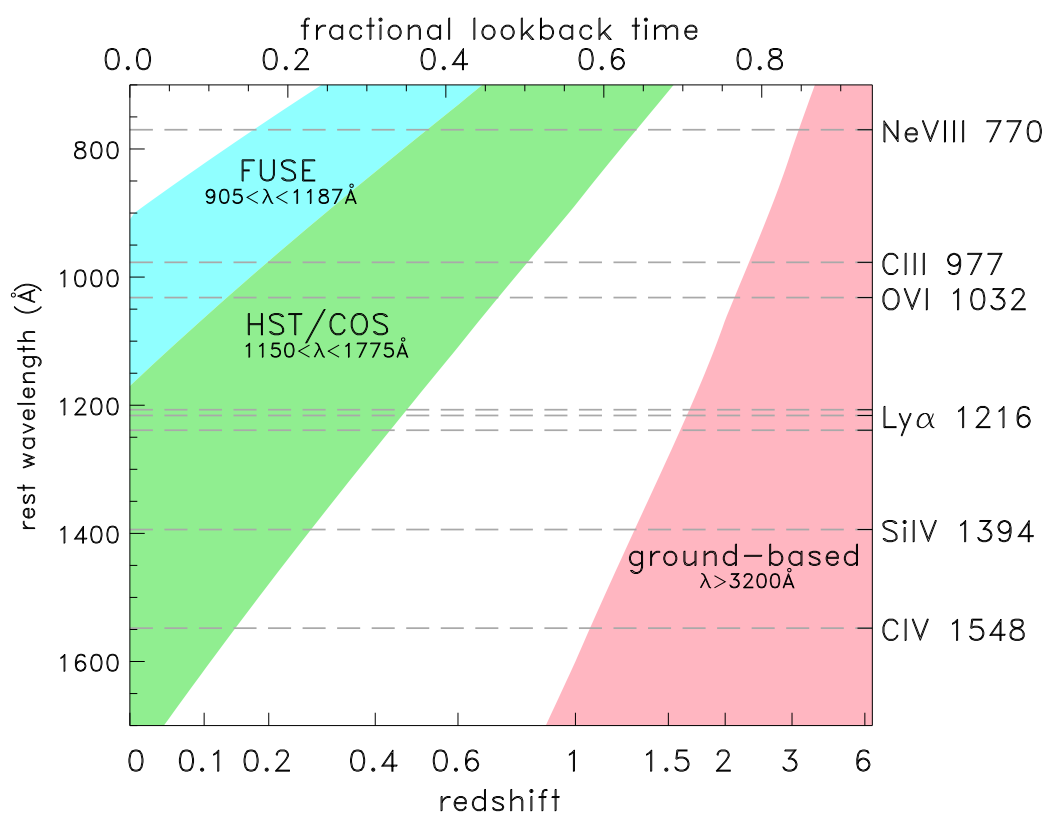

FIGURE 2. Diagram showing redshifts and lookback times probed by various UV lines studied by HST, FUSE, and ground-based telescopes (Keck, VLT). Studying the "near-UV desert" (diagonal white band between $z=0.5-1.5$ ) poses observational challenges, owing to the lack of bright target AGN and to the low quantum efficiency of current NUV detectors.

damped Ly $\alpha$ absorber). There are also cosmological applications. With over 10,000 Ly $\alpha$ forest absorbers at $z<0.4$, we can derive power spectra, $P(k)$, in both absorption and transmission. Continuing work in the GTO program, we hope to study the evolution of the He II reionization process between $z=2.8-3.2$.

\section{STRATEGIES FOR THE NEXT-GENERATION UVO MISSIONS}

As noted earlier, we anticipate an exciting 5-10 years of UV spectroscopic science with COS and Hubble. However, astronomers are already planning the next generation satellite missions, in nearly all wavelength bands. This is prudent, given the long lead times and budgetary constraints. Even in the best case for NASA astrophysics funding, both UV/O and X-ray space astronomy are likely looking at a ten-year gap, extending from perhaps 2016 to 2025. Let us therefore advocate to NASA how to fill this gap.

First of all, our field of ultraviolet-optical space astronomy should be proud of what Hubble, FUSE, and Galex have achieved. There may be some complacency that Hubble could last indefinitely, but it will not. Astronomers at STScI and in the general community are already proposing new ideas for missions, including a flagship mission, ATLAS$T$ (in $8 \mathrm{~m}$ or $16 \mathrm{~m}$ varieties) and moderate-cost missions such as the Baryon Structure Probe. NASA has also funded a study of a 4-meter mission (THEIA) for combined widefield UV imaging, UV spectroscopy, and extrasolar planet characterization. 
The major issues facing our discipline include: a limited ( $\sim 1 \mathrm{~B})$ annual budget for the Astrophysics Division, an uncertain set of priorities coming from the 2009-2010 Decadal Survey, and many uncompleted projects (JWST, SOFIA, JDEM, IXO, LISA). Below is a personal list of major science drivers that might motivate NASA and our astrophysics profession to consider a UV/O flagship mission, followed by a list of desired capabilities. Related issues were discussed in the 1999 White Paper on the Space Ultra-Violet Optical Observatory (SUVO) from the UVOWG Committee Report: "The Emergence of the Modern Universe: Tracing the Cosmic Web" (http://arxiv.org/abs/astro-ph/9907101).

\section{Top Science goals for a Major UV/Optical Mission}

- Map the cosmic web and intervening galactic halos (AGN to $V=20-21^{\mathrm{m}}$ )

- Study galaxy assembly and stellar populations with deep UV/O imaging

- Perform precision cosmology to $1 \%$ (Cepheids, weak lensing)

- Characterize extra-solar planets (images, spectra, occulter)

What are the Desired UV Capabilities?

- High sensitivity (large $A_{\text {eff }}$ spectrograph in the FUV)

- Good spectral resolution (10 $\mathrm{km} \mathrm{s}^{-1}$ with $3 \mathrm{~km} \mathrm{~s}^{-1}$ mode desirable)

- High spatial frequency of targets on the sky (AGN at 1 per arcmin)

- Wide-field imaging (10 arcmin and > 20 arcmin if possible)

- Wide-field emission-line mapping (to 10 photon line-units)

- Ability to conduct time-domain studies

\section{A Balanced Suite of Missions and Launch Intervals for NASA}

Looking forward to the next 20 years, what are the best strategies for achieving some of the science drivers afforded by UV/Optical telescopes? Foremost in the scientific argument are the intrinsic UV capabilities. As a result of atomic physics (cross sections, resonance line energies, physical diagnostics), the ultraviolet band provides the most sensitive probes of matter in the form of atoms, ions, and molecules $\left(\mathrm{H}_{2}, \mathrm{HD}, \mathrm{CO}\right.$, etc.) For instance, the Ly $\alpha$ line of hydrogen is $10^{6}$ times more sensitive to column density, $\mathrm{N}_{\mathrm{HI}}$, than $21-\mathrm{cm}$ emission. UV resonance lines of important ion stages of heavy elements $(\mathrm{C}, \mathrm{N}, \mathrm{O}, \mathrm{Si}, \mathrm{S}, \mathrm{Fe}$ ) fall in the far-UV band. However, other wavelength bands all have strong rationales for how they are well-suited as astrophysical probes owing to dustobscuration, redshifting, or high-energy processes. So, what is our best course of action? First, we need to come together on the scientific rationale for a large-aperture, costeffective UV/O space telescope to do the science that is still beyond the capabilities of Hubble. This study is now underway for ATLAS-T (see Postman paper). For the intermediate term, we should consider what science can be done with smaller missions, with focused tasks and conscious tradeoffs:

- Combine UV spectroscopy with planet-finding (or have dedicated mission?)

- Separate mission for faint emission-line mapping?

- Separate FUSE band $(\lambda<1150 \AA)$ from STIS/COS band $(\lambda>1150 \AA)$ ? 
We also need to be reasonable about cost, if we wish to have regular access to space. This probably means only one astrophysics flagship mission per decade. Table 2 illustrates a balanced program of missions (small, medium, large) with costs and launch intervals that fit under the current NASA-Astrophysics cost umbrella. Averaged over a decade, they total $\$ 760 \mathrm{M} /$ year, allowing additional funds for mission operations, data analysis, technology development, and other important programs (such as theory and lab astrophysics).

TABLE 2. A Balanced Spaceflight Program

\begin{tabular}{lrrr}
\hline Mission & Total Cost & Interval & Annual Cost \\
\hline Flagship & $\$ 4 \mathrm{~B}$ & $10 \mathrm{yrs}$ & $\$ 400 \mathrm{M} / \mathrm{yr}$ \\
Probe & $\$ 750 \mathrm{M}$ & $5 \mathrm{yrs}$ & $\$ 150 \mathrm{M} / \mathrm{yr}$ \\
MidEx & $\$ 360 \mathrm{M}$ & $3 \mathrm{yrs}$ & $\$ 120 \mathrm{M} / \mathrm{yr}$ \\
SmEx & $\$ 180 \mathrm{M}$ & $2 \mathrm{yrs}$ & $\$ 90 \mathrm{M} / \mathrm{yr}$ \\
\hline Total & & & $\$ 760 \mathrm{M} / \mathrm{yr}$
\end{tabular}

However, NASA rarely operates with long-range budgets, as they are constantly facing cost overruns and budget shortfalls. One of the important tasks for us, and for NASA administrators, is to convince everyone - the public, our profession, and Congress - that space astrophysics is an exciting area in which to invest. The problems are compelling, they excite the public, they stimulate education and technology, and they deserve more than the $\$ 1 \mathrm{~B} /$ year currently devoted to the Astrophysics Division of NASA. A rising tide will raise all ships (and many wavelength bands).

\section{ACKNOWLEDGMENTS}

Travel funding to Annapolis was generously provided by NASA/GSFC. Our group's research support at the University of Colorado for UV studies of the IGM and Galactic halo gas comes from COS grant NNX08-AC14G and STScI spectroscopic archive grants (AR-10645.02-A and AR-11773.01-A). I would also like to express appreciation for many years of NASA support from the FUSE data analysis program, which enabled so many new ISM and IGM discoveries through far-UV spectroscopy.

\section{REFERENCES}

1. Cen, R., \& Ostriker, J. P. 2006, ApJ, 650, 560

2. Collins, J. A., Shull, J. M., \& Giroux, M. A 2004, ApJ, 605, 216

3. Collins, J. A., Shull, J. M., \& Giroux, M. A 2007, ApJ, 657, 271

4. Danforth, C. W., \& Shull, J. M. 2008, ApJ, 679, 194

5. Davé, R., et al. 2001, ApJ, 552, 473

6. Froning, C. S., \& Green, J. C. 2008, ApSS, on-line

7. Hallman, E. J., et al. 2007, ApJ, 671, 27

8. Morse, J., et al. 1998, SPIE, 3356, 361

9. Savage, B. D., et al. 2005, ApJ, 626, 776

10. Stocke, J. T., et al. 2006, ApJ, 641, 217

11. Tripp, T. M., et al. 2008, ApJS, 177, 39

12. Weymann, R. J., et al. 1998, ApJ, 506, 1 